\title{
Nanoestructuras metálicas y su resonancia de plasmones superficial
}

\author{
José Luis Fraga Almanza \\ josefraga@uadec.edu.mx \\ Programa de Posgrado de Doctorado en Ciencia \\ y Tecnología de Materiales, \\ Facultad de Ciencias Químicas, \\ Universidad Autónoma de Coahuila \\ Saltillo-México.
}

Carlos Eduardo Rodríguez García crodriguezgarcia@uadec.edu.mx Facultad de Ciencias Físico Matemáticas, Universidad Autónoma de Coahuila Saltillo-México.

Elmer Cruz Mendoza elmer.cruz@uabc.edu.mx

Facultad de Ciencias, Universidad Autónoma de Baja California, Ensenada-México.

Marco Antonio García Lobato marco.garcia@uadec.edu.mx Facultad de Ciencias Químicas Universidad Autónoma de Coahuila Saltillo-México.

Juan Segura Sosa juan_segura@uadec.edu.mx Facultad de Ciencias Físico Matemáticas, Universidad Autónoma de Coahuila Saltillo-México.

Erika Martínez Sánchez erika.martinez@uadec.edu.mx Facultad de Ingeniería Universidad Autónoma de Coahuila

\section{RESUMEN}

Arteaga-México

Este artículo es una breve revisión divulgativa sobre las nanoestructuras metálicas de diferentes geometrías, su respuesta óptica de resonancia de plasmones superficial y algunas aplicaciones seleccionadas. Las nanoestructuras metálicas de entre 1-100 nm presentan la interesante característica de resonancia de plasmones superficial la cual se 
induce por la interacción con luz. Como nanoestructuras más comunes podemos encontrar las nanopartículas esféricas de Plata $(\mathrm{Ag})$ y Oro $(\mathrm{Au})$, cuyos plasmones superficiales son muy conocidos en la literatura. La propiedad plasmónica puede generar una variedad de aplicaciones en la nanociencia y nanotecnología en estas nanopartículas metálicas. Con relación a esto último, en este trabajo, citamos algunas que consideramos importantes que son: mitigación de bacterias utilizando nanopartículas de Ag, sensores de glucosa utilizando nanopartículas tipo dona de óxido de Níquel y mejoramiento de fototerapia para tratamientos de cáncer con nanopartículas de Au.

Palabras clave: nanoestructuras; nanopartículas; plasmones; resonancia-superficial. 
Fraga Almanza y Rodríguez García...

\title{
Metallic nanostructures and their Surface plasmons resonance
}

\begin{abstract}
In this paper we present a short divulgative revision related to metallic nanostructres with diverse geometry, their optical response of localized surface plasmons resonance and some selected aplications. These metallic nanostructures with sizes in the range of 1-100 $\mathrm{nm}$, showed an interesting localized surface plasmons resonance phenomenon which is presented for interacting with ligth. The most common nanostructures are Silver (Ag) and Gold $(\mathrm{Au})$ spherical nanoparticles, which surface plasmons are very well know in the literature. Thus, the plasmonic property generates several aplications in nanocience and nanotechnology. Regarding the latter, in this work we cite the most representatives references in the topics such as: bacteria mitigation by using Ag nanoparticles, glucosa sensing by utilizing nanodonougths of Nickel oxide, and the improvement of cancer phototerapy with Au nanoparticles.
\end{abstract}

Keywords: nanoestructures; nanoparticles; plasmons; surface-resonance.

Artículo recibido: 15 noviembre. 2021 Aceptado para publicación: 10 diciembre 2021 Correspondencia: josefraga@uadec.edu.mx Conflictos de Interés: Ninguna que declarar 


\section{INTRODUCCIÓN}

A mediados del siglo pasado comenzó una carrera por la miniaturización de los dispositivos tecnológicos. Esto, ha permitido un fantástico desarrollo en áreas tales como la electrónica, la microelectrónica y la nanoelectrónica (Cherie R. Kagan y otros, 2016), en donde los sistemas son cada vez más pequeños (por lo general formados por nanoestructuras y nanopartículas). Adicionalmente, trabajos teóricos y experimentales, muestran que dicha reducción en el tamaño de las estructuras da lugar a cambios en las propiedades de la materia, lo cual abre nuevos paradigmas en la investigación científica dentro del área de la Nanociencia y Nanotecnología (Bayda, Adeel, Tuccinardi, Cordani, \& Rizzolio, 2019).

Diversos autores, definen las nanoestructuras como partículas con dimensiones de 1 a 100 $\mathrm{nm}$, de las cuales, se sabe, sus propiedades dependen fuertemente del tamaño (Boholm \& Arvidsson, 2015). En este sentido, las nanopartículas son nanoestructuras cuyas geometrías van desde las sencillas nanoesferas, nanocubos, nanobarras, hasta otras con geometrías más complejas y curiosas como nanoestrellas, nanoflores, nanodonas, por mencionar algunas (Loiseau, y otros, 2019). Cabe destacar, que tales nanopartículas poseen potenciales aplicaciones en el desarrollo de tecnología inovadora: por ejemplo, las nanoesferas de Ag (Plata) poseen propiedades antimicrobianas y pueden ser utilizadas en vendajes médicos (C. Cardoso, 2016). Así mismo, las nanodonas de NiO (óxido de Níquel) son empleadas como componentes de un sensor electroquímico para la medición glucosa con muy buenos resultados (Ahmad, y otros, 2020), comparados con los de un sensor comercial. Por tanto, el estudio e investigación de las nanoestructuras es un campo vivo en las nanociencias que amplía la posibilidad de ser utilizadas en la nanotecnología para beneficio de la sociedad.

En la Figura 1 se exhibe una imagen donde se ilustran este tipo de nanoestructuras. A pesar de que existe un gran número de nanoestructuras estudiadas con diferentes geometrías, recientes investigaciones muestran que geometrías muy "raras" como nanodonas y nanocintas de Moebius pueden existir experimentalmente (Geng, y otros, 2019; Ahmad, y otros, 2020). No obstante, cabe mencionar que hace falta explorar con mayor profundidad las propiedades de este tipo de nanoestructuras exóticas, ya que como sabemos, un mayor entendimiento de estas permitiría explotarlas en posibles aplicaciones tecnológicas. 
Figura 1. Micrografías electrónicas de barrido de nanoestructuras y nanopartículas con diferentes geometrías. A) esferas, B) cubos, C) triángulos, D) octaedros, E) flores, F) barras, G) arroces, H) barras, I) zanahorias y J) Alambres (Imagen adquirida de Hongyang Liang y col, 2015)
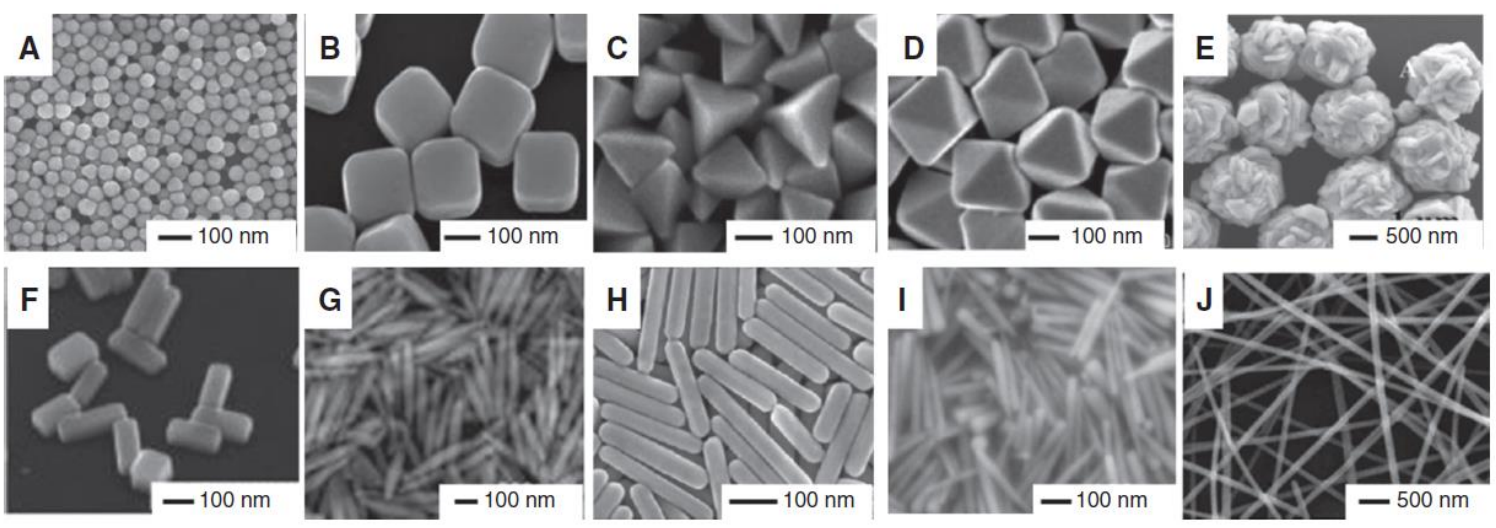

Por otro lado, un efecto que ha cobrado relevancia desde la década de los 90 es el de la Resonancia de Plasmones Localizados de Superficie (RSPL), los cuales son excitaciones colectivas de la densidad carga que se presentan en sistemas con altas densidades electrónicas, tales como nanopartículas y nanoestructuras metálicas. Por ejemplo, podemos mencionar estudios realizados en estructuras construidas a partir de metales nobles como la Ag (Plata) y el Au (Oro) (Santillán, 2013). El estudio y entendimiento de las propiedades del RSLP sobre estas nanoestructuras ha abierto la puerta a un gran número de interesantes aplicaciones tecnológicas, como, por ejemplo: fabricación de sensores ultrasensibles (Loiseau y otros, 2019), fuentes de radiación térmica localizada para eliminar células cancerígenas en tejido vivo (Swami Muddineti y otros, 2015), celdas solares con mayor eficiencia y el mejoramiento de espectroscopias electrónicas (Haume, K. Rosa y otros, 2016).

Dada la relevancia de las propiedades de las nanopartículas metálicas en distintas áreas de la ciencia, en este trabajo se presenta, a manera de divulgación, una breve revisión sobre propiedades importantes que algunas nanoestructuras presentan como resultado de su forma y tamaño. Particularmente, se explorarán los avances generados por la comunidad científica sobre la excitación y manipulación del RSLP en nanoestructuras con distintas geometrías. La intención, es elucidar sobre aquellos sistemas con tamaños y formas que han sido poco explorados y los cuales representarían los problemas a resolver en un futuro. 


\section{METODOLOGIA}

La investigación presente es una revisión de algunos artículos seleccionados reportados en Google Académico en revistas indizadas en Journal of citation of reports (JCR) u otros índices como Latindex. La metodología usada para tal búsqueda fue mediante los siguientes criterios: 1) frases claves en inglés como "metallic nanostructures", "nanoparticles and geometry". "localized surface plasmon resonance”; 2) Análisis de la información centrado en las nanoestructuras metálicas, enfocandose en aspectos tales como: (i) geometrías, (ii) respuesta plasmónica y (iii) posibles aplicaciones; 3) Se indagó sobre nanoestructuras poco estudiadas como lo son las nanodonas de Oro y de óxido de Níquel.

Además, para singularizar la revisión presentamos las nanopartículas de distintas geometrías más comunes tomando en cuenta los metales: Plata y Oro.

\section{RESONANCIA PLASMÓNICA SUPERFICIAL LOCALIZADA}

Es bien sabido que materiales conductores tales como los metales, poseen una alta densidad electrónica $\left(10^{22}\right.$ electrones $\left./ \mathrm{cm}^{3}\right)$ constituida por los electrones $\left(e^{-}\right)$de la banda de conducción, los cuales pueden moverse libremente dentro del material. Así mismo, se sabe que al interaccionar con campos electromagnéticos se generan oscilaciones de la densidad de carga y a cuya cuantización se le da el nombre de plasmón. Cuando los sistemas son infinitos, en principio, los electrones pueden moverse libremente sin ninguna restricción, como por ejemplo cuando la corriente eléctrica pasa a través de un cable largo que es perfecto conductor, los electrones que atraviesan por una sección transversal por unidad de tiempo se mueven libremente sin encontrar condiciones que modifiquen su dinámica. Sin embargo, esto no sucede en sistemas confinados tales como las nanoestructuras metálicas cuyos tamaños rondan tamaños de 1 a 100 nm. En este caso, las propiedades de transporte electrónico se modifican considerablemente ya que el movimiento de los electrones se encuentra restringido por el tamaño del sistema, así como por las condiciones de frontera de este. Consecuentemente, los plasmones también se ven afectados, ya que ahora se convierten en modos resonantes definidos por la densidad de carga de la nanoestructura y confinados por la superficie de esta. A esta clase de modos colectivos se les conoce como el nombre de Resonancia del Plasmón Localizado de Superficie (RSPL). Una característica interesante de los RSPL es que pueden ser excitados con luz (Mendoza Herrera, 2018), esto los convierte, junto con las 
nanopartículas metálicas, en potenciales candidatos para el desarrollo de múltiples aplicaciones en nanofotónica; al área encargada de estudiar y explorar las propiedades de los plasmones se conoce como plasmónica.

Para entender la excitación de los RSPL por medio de la luz considere lo siguiente: Suponga una nanopartícula metálica (de forma arbitraria), la cual se encuentra bajo la acción de una onda electromagnética caracterizada por un campo eléctrico $\boldsymbol{E}$ transversal a la dirección de propagación de la onda. Entonces, la densidad de carga de la nanopartícula se acoplará con dicho campo eléctrico, de manera que la nanoestructura sufrirá una polarización de carga, tal como se muestra en la figura 2. Sin embargo, como la dirección de $\boldsymbol{E}$ varía como función del tiempo, la polarización de la nanopartícula también cambiará y de esta manera la densidad de carga de todo el sistema entrará en resonancia dando lugar a plasmones caracterizados por una frecuencia $\omega_{p}$, a la cual se le conoce como la frecuencia de resonancia del plasmón.

Figura 2. Esquema de cómo la luz como onda electromagnética con su campo eléctrico E incide sobre dos nanopartículas metálicas de distinta geometría y hace que oscile la nube electrónica en cada nanoestructura.

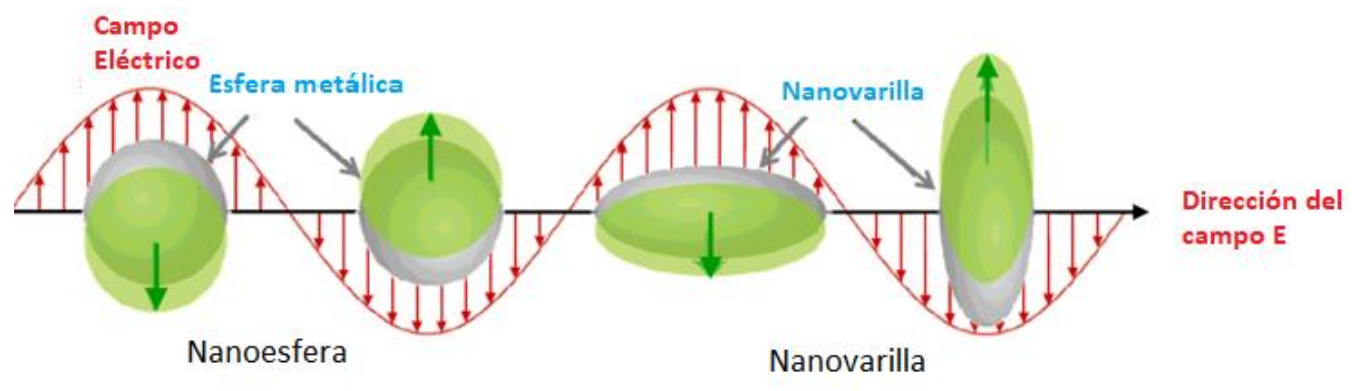

La imagen fue adaptada de referencia (Álvares-Puebla, 2021).

La RSLP generalmente se puede detectar por medio de la técnica de espectroscopia de Ultravioleta-Visible mediante un espectrofotómetro, el cual mide la extinción (atenuación) de la luz al transmitirse por una muestra que contiene nanoestructuras o nanopartículas. Las nanoestructuras pueden estar disueltas en solución en diferentes solventes. En la Figura 3 se proyectan fotografías de nanopartículas de Au, con diferentes diámetros (20 nm y $100 \mathrm{~nm}$ ) los cuales logran una respuesta óptica distinta debido a la RSPL (NSF Center for Sustainable Nanotechnology, 2021). También en el tercer recipiente tenemos hojuelas de Au macroscópico, estas soluciones presentan distintos 
colores, y el material sólido es bien conocido que tiene un color amarillo brillante, lo que conlleva diferentes valores de extinción de la luz.

Figura 3. Recipientes que contienen nanopartículas esféricas de $\mathrm{Au}$ de diferentes diámetros, $20 \mathrm{~nm}$ y $100 \mathrm{~nm}$. En el tercer recipiente notamos hojuelas de Au a nivel macroscópico.

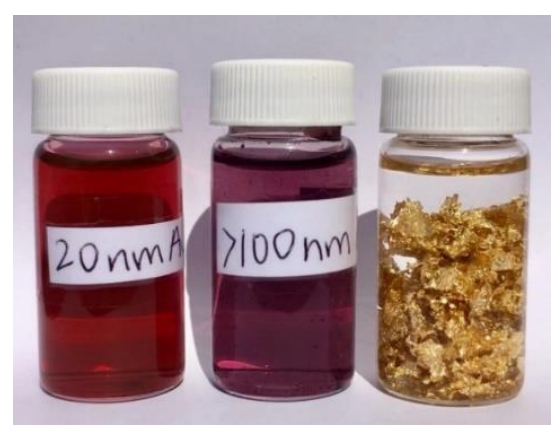

La imagen fue adquirida de (NSF Center for Sustainable Nanotechnology, 2021).

Como notamos, analizar las propiedades de la RSLP en nanopartículas nos conlleva a utilizar el concepto de la extinción. Supongamos que tenemos una muestra de espesor X y con una concentración porcentual de nanopartículas esféricas de Oro (Au), por la cual incide luz con intensidad inicial $\mathrm{I}_{0}$, de la muestra sale luz con intensidad $\mathrm{I}$, con $\mathrm{I}<\mathrm{I}_{0}$ por lo que parte de la luz se quedó en la muestra, digamos una cantidad $\beta$. En este esquema de la Figura 4, el detector sería un espectrofotómetro que medirá la extinción en un rango de longitudes de onda de luz. A la fracción de luz removida del haz incidente se le denomina extinción $(\beta)$.

Figura 4. Esquema de una muestra con nanopartículas de $\mathrm{Au}$ donde incide luz con intensidad $\mathrm{I}_{0}$ la cual es absorbida, para luego detectar una saliendo de la muestra luz con intensidad I.

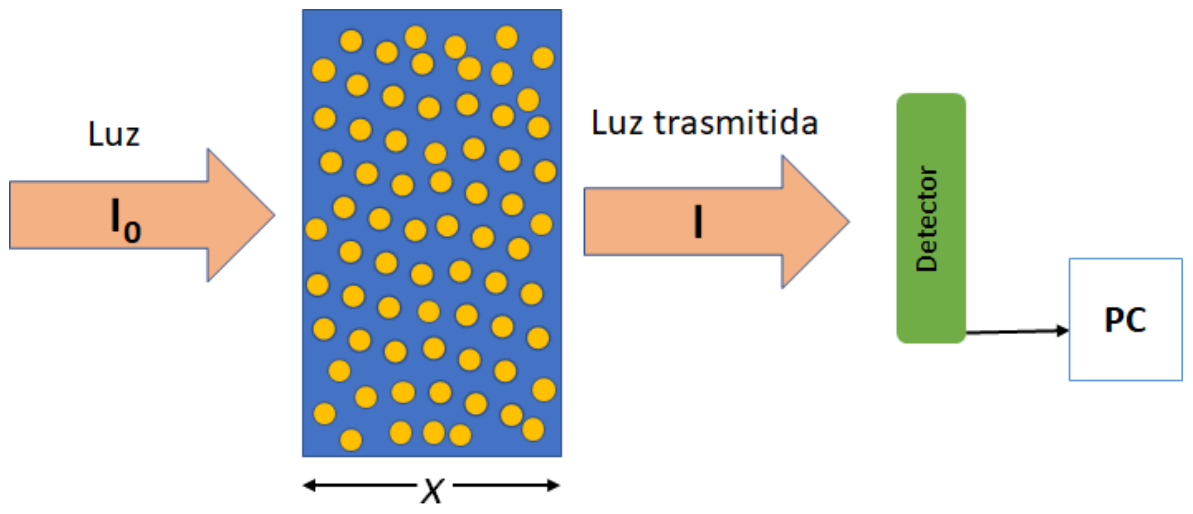

Nanopartículas de Au

Fuente: Elaboración propia. 
La ecuación para la Ley de Lambert Beer nos provee una forma de fundamentar la extinción la cual es:

$$
I=I_{0} \exp (-\beta X)
$$

Donde I es la intensidad a la salida de la muestra con nanopartículas, Io la intensidad inicial, $\beta$ la extinción y $X$ la longitud de la muestra. La extinción $\beta$ incluye dentro de su medición la luz absorbida y dispersada. Un ejemplo de la extinción óptica para nanopartículas que tienden a lo esférico de Ag lo encontramos en la Figura 5, a lado derecho una imagen de microscopia electrónica de Transmisión de estas nanopartículas. Se observa un pico prominente de extinción en $420 \mathrm{~nm}$ aproximadamente, la imagen y el espectro fue adquirida de la referencia (Reyes Gómez, y otros, 2018).

Figura 5. (izquierda) Espectro de extinción de nanopartículas de Ag y (derecha) nanopartículas de Ag vistas por microscopia electrónica de transmisión obtenidas de (Reyes Gómez, y otros, 2018).

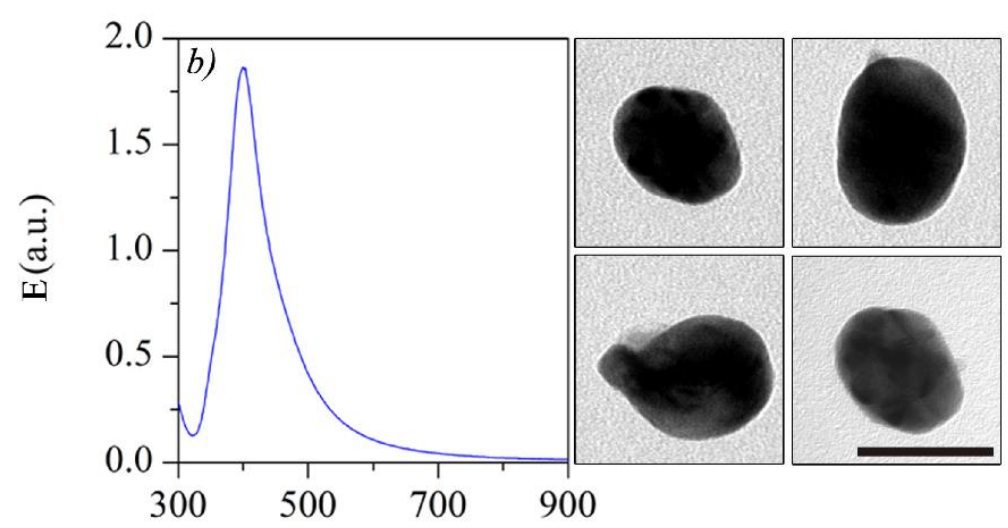

Esta extinción detectada es debida a esa RSLP, dado que cierta energía de la luz se absorbe y dispersa para poder poner en resonancia la nube electrónica de esas nanoestructuras. Tal respuesta de RSLP puede ser usada para diferentes aplicaciones, algunas de estas aplicaciones más comunes de las nanoestructuras metálicas se explicarán brevemente en la siguiente sección.

\section{APLICACIONES DE LAS NANOESTRUCTURAS METÁLICAS}

\section{Acción bactericida de las Nanopartículas de Ag}

Una de las aplicaciones más expandidas de las nanopartículas de Plata (Ag) es su utilización como bactericida. En (Gómez Quintero, Arroyo Ornelas, Hernández Padrón, \& Acosta Torres, 2013). describen que las nanopartículas de Ag son capaces de inhibir el 
crecimiento de la bacteria Escherichia coli (E. Coli), la cual comúnmente se encuentra en el intestino. Otra aplicación interesante es el ataque que tienen las nanopartículas de plata con la bacteria Candida Albicans. Tal bacteria tiene un crecimiento en las prótesis dentales de resina acrílica, pero cuando nanopartículas de Ag son embebidas se inhibe el crecimiento de esa bacteria (De Jong, Jansen, van der ven, \& van der Zee, 2013). Así mismo, se utilizan nieblas de nanopartículas de Ag en los hospitales para remover bacterias superresistentes en los sistemas de ventilación y/o cuartos de los pacientes. En la Figura 6, se presentan una imagen de microscopia electrónica de transmisión de nanopartículas de Plata con tamaños en el rango de 10-70 nm.

Figura 6. Micrografía electrónica de transmisión de nanopartículas de plata que tienen propiedades bactericidas,

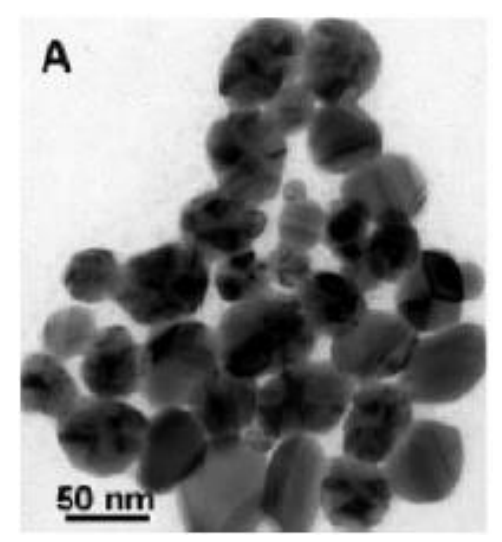

Fotografía del trabajo de (Pal, Kyung Tak, \& Myong Song, 2007).

Incluso otros trabajos muy recientes, están explorando el uso incipiente de las nanopartículas de Ag como viricida, es decir, desactivar virus de superficies, como lo es el Covid-19 (Chang, y otros, 2021). Lo que, sin duda, será una de las aplicaciones más fascinantes que apoyen a la nanomedicina para erradicar el virus SARS-Cov 2 en esta pandemia mundial.

\section{Nanopartículas de Au para fototerapia contra el Cáncer}

El cáncer es una de las enfermedades más importantes que tiene una alta mortandad y que el número de pacientes diagnosticados está incrementando rápidamente en la actualidad (Haume, y otros, 2016). Diversos canceres cuando son detectados en etapas primarias pueden abordarse por medio de cirugía o fototerapia. Esta última técnica, posee un $50 \%$ de eficacia para la eliminación de tumores cuando son detectados a tiempo (Haume, y 
otros, 2016). La fototerapia es el uso de pulsos de luz o radiación continua sobre algún órgano con la finalidad de erradicar células cancerosas, comúnmente se utiliza para ellos luz de alta energía como suelen ser los rayos-X o rayos gamma. No obstante, dada las comorbilidades o la localización de los tumores, en ocasiones no es posible aplicar esta técnica de forma efectiva. Una de las soluciones estudiadas recientemente (Haume, y otros, 2016) es el uso de nanopartículas metálicas, las cuales pueden absorber esta alta energía y transferirla de forma localizada al tumor o células de cáncer. Otra manera de atacar a un tumor canceroso es hacer daño localmente para provocar un cierre de flujo de nutrientes que mantienen el aumento del tumor. Ciertas nanopartículas que se han explorado potencialmente para tal fin son las de Oro (Au) debido a su buena biocompatibilidad, sencilla funcionalización en las superficies donde se encuentran las células cancerosas y su facilidad de síntesis (Swami Muddineti, Ghosh, \& Biswas, 2015). La presencia de nanopartículas de Au en los órganos donde se encuentran estas células dañinas incrementa la razón de dosis recibida por medio de la fototerapia (Swami Muddineti, Ghosh, \& Biswas, 2015). Por las razones anteriores, es muy posible que las encontremos en los tratamientos médicos contra el cáncer en los próximos años.

\section{Nanodonas para la detección de glucosa}

En (Rai, Nayak, \& Roy Barman, 2015), estudiaron experimentalmente la deposición por láser de nanoestructuras de $\mathrm{Au}$ con geometrías complejas interesantes tipo nanodonas. Cabe mencionar, que para esta geometría existen pocos estudios, y en su mayoría son experimentales. Estas nanodonas se presentan en superficies metálicas y son generadas por la presencia de gases como Argón y Nitrógeno, al formar burbujas durante el proceso. Pero ¿qué tienen de interesantes estas nanoestructuras? En otros estudios, pero con óxidos metálicos, por ejemplo, el óxido de Níquel, la formación de nanodonas ha servido para aplicaciones en detección de glucosa. Lo que permite la elaboración de sensores basados en las nanodonas de $\mathrm{NiO}$ con muy buena detección de la glucosa en el límite bajo de detección de $1.4 \mu \mathrm{M}$ de glucosa comparado con los sensores comerciales (Ahmad, y otros, 2020). Por tanto, en los siguientes años esperemos encontrar sensores que utilicen estas interesantes y complejas estructuras. En la Figura 7 se presenta una imagen de micrografía electrónica de barrido obtenida de la publicación de (Ahmad, y otros, 2020), donde se pueden observar las nanodonas de $\mathrm{NiO}$. 
Figura 7. Imágenes de microscopia electrónica de barrido de nanoestructura tipo dona del material $\mathrm{NiO}$ utilizadas en los sensores de glucosa

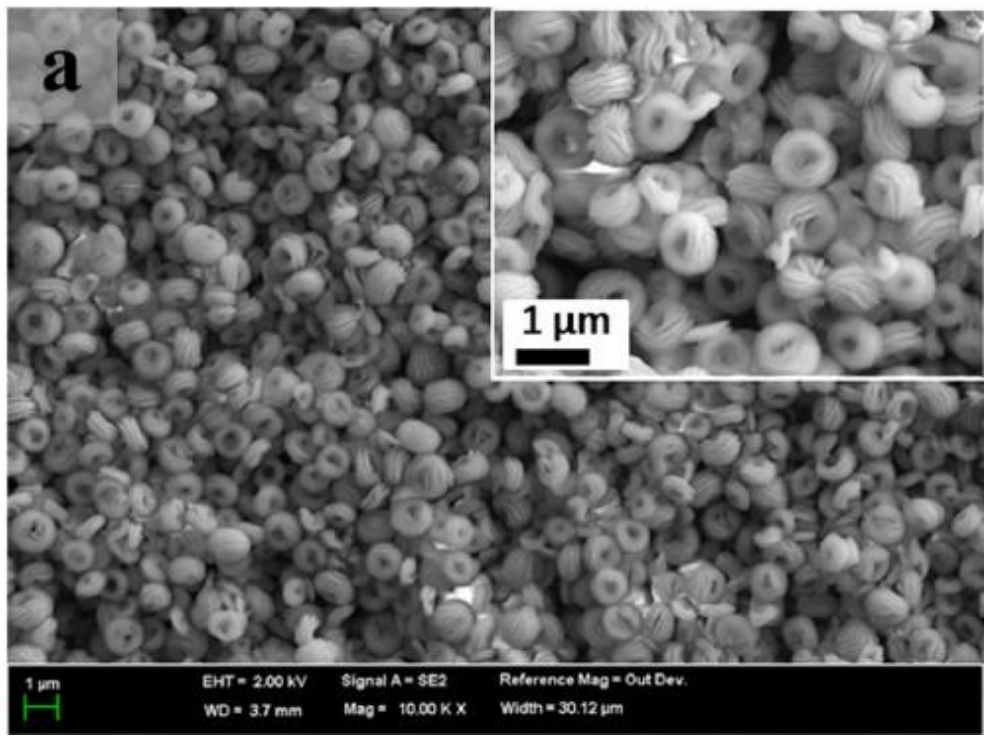

La imagen se adquirió de (Ahmad, y otros, 2020).

A pesar de que existen diferentes aplicaciones que utilizan la RSLP de las nanoestructuras metálicas, aún es un tema intensamente activo en la investigación científica hoy en día. Por lo que, esperamos en los años futuros nazcan otros desarrollos nanotecnológicos que utilicen la RSLP como una ventaja para el progreso en la ciencia y tecnología de los nanomateriales.

\section{CONSIDERACIONES FINALES}

1) La Resonancia de Plasmones de Superficies Localizados (RSLP) como fenómeno óptico en nanoestructuras y nanopartículas metálicas es un importante campo en la nanociencia y nanotecnología de materiales hoy en día. La búsqueda de nuevas geometrías en nanoestructuras metálicas se encuentra dentro de las prospectivas de la RSLP debido a que esta propiedad está íntimamente relacionada a las diferentes formas en las nanopartículas. Tal respuesta óptica podría contribuir al desarrollo de nuevas aplicaciones.

2) Los metales más comunes que han sido estudiados para esta respuesta plasmónica son la Plata y el Oro, y las geometrías más estudiadas son la esfera por simplicidad y minimización de la energía. Aunque, la investigación de este efecto óptico no se limita a los metales se pueden encontrar la detección de la RSLP en semiconductores de 
óxidos metálicos. Por lo que, es interesante que se siga investigando ciencia básica y aplicada en este el campo de la RSLP.

3) A pesar de que existen diferentes aplicaciones que utilizan la RSLP de las nanoestructuras metálicas, aún es un tema intensamente activo en la investigación científica hoy en día. Por lo que, esperamos en los años futuros nazcan otros desarrollos nanotecnológicos que utilicen la RSLP como una ventaja para el progreso en la ciencia y tecnología de los nanomateriales.

4) Finalmente, las aplicaciones más exploradas recientemente son la erradicación de bacterias y superbacterias resistentes con nanopartículas de Ag, el mejoramiento en la eliminación de células cancerosas por el incremento de la razón de dosis recibida por medio de la fototerapia en la presencia de nanopartículas de Au, y la construcción de sensores hechos con nanodonas de $\mathrm{NiO}$ para detección de glucosa.

\section{REFERENCIAS BIBLIOGRAFICAS}

Ahmad, R., Khan, M., Rizwan Khan, M., Tripathy, N., R. Khan, M., Mishra, P., . . . Khosla, A. (2020). Nano-donuts shaped nickel oxide nanostructures for sensitive non-enzymatic electrochemical detection glucose. Springer-Verlag $\mathrm{GmbH}$ Germany, 6.

Álvares-Puebla, R. Á. (1 de 11 de 2021). Centre Tecnológic de la Química de Catalunya. Obtenido de http://ctqcquimicasostenible.blogspot.com/2016/01/los-avances-dela-ciencia-por-el-prof.html

Bayda, S., Adeel, M., Tuccinardi, T., Cordani, M., \& Rizzolio, F. (2019). The History of Nanoscience and Nanotechnology: From Chemical-Physical Applications to Nanomedicine. Molecules, 15.

Boholm, M., \& Arvidsson, R. (2015). A Definition Framework for the Terms Nanomaterial. Springer Science, 16.

C. Cardoso, P. (2016). Nanopartículas de plata: obtención, utilización como antimicrobiano e impacto en el área de la salud. 10.

Chang, S.-Y., Huang, K.-Y., Chao, T.-L., Kao, H.-C., Pang, Y.-H., Lu, L., . . Yang, P.-C. (2021). Nanoparticle composite TPNT1 is effective against SARS-Cov-2 and influenza viruses. Nature Scientifics Reports, 13. 
De Jong, W., Jansen, E., van der ven, L., \& van der Zee, M. (2013). Systemic and immunotoxicity of silver nanoparticles in an intravenous 28 days repeated dose toxicity study in rats. Biomaterials, 12.

Geng, Z., Xiong, B., Wang, L., Wang, K., Ren, M., Zhang, L., . . . Yang, Z. (2019). Moebius strips of chiral block copolymers. Nature Comunications, 9.

Gómez Quintero, T., Arroyo Ornelas, M., Hernández Padrón, G., \& Acosta Torres, L. (2013). Nanopartículas de plata: Aplicaciones biomédicas. QUIMICA HOY Chemistry Sciences, 12.

Haume, K., Rosa, S., Grellet, S., A. Śmiałek, M., Butterworth, K., Solov’yov, A., . . . Mason, N. (2016). Gold nanoparticles for cancer radiotherapy: a rewiew. Springer , 20.

Loiseau, A., Asila, V., Boitel-Aullen, G., Lam, M., Salmain, M., \& Boujday, S. (2019). Silver-Based Plasmonic Nanoparticles for and Their Use in Biosensing . Biosensors, 40.

Mendoza Herrera, L. (2018). Estudio de la respuesta óptica de nanoestructuras plasmónicas complejas. Argentina.

NSF Center for Sustainable Nanotechnology. (1 de 11 de 2021). Obtenido de https://sustainable-nano.com/about/

Pal, S., Kyung Tak, Y., \& Myong Song, J. (2007). Does the Antibacterial Activity of Silver Nanoparticles Depend on the Shape of the Nanoparticle? A Study of the Gram-Negative Bacterium Escherichia coli. APPLIED AND ENVIRONMENTAL MICROBIOLOGY, 9.

Rai, A., Nayak, J., \& Roy Barman, S. (2015). Nano-donuts on metal surfices. Elsevier, 6.

Reyes Gómez, F., G. Rubira, R., A. Camacho, S., S. Martin, C., R. da Silva, R., L. Constantino, C., . . . Mejía Salazar, J. (2018). Surface Plasmon Resonances in Silver Nanostars. Sensors, 9.

Santillán, J. (2013). Estudios de las propiedades ópticas de materiales nanoestructurados y aplicaciones. Argentina.

Swami Muddineti, O., Ghosh, B., \& Biswas, S. (2015). Current trends in using polymer coated gold nanoparticles for cancer therapy. Elsevier, 16. 